\title{
SPLENIC CYST
}

\author{
Larissa L. Garcia*, Fernanda. L. Lomanto*, Felipe Simões da Rocha Mata**, Dilton Mendonça*** \\ Corresponding author: Larissa Latrilha Garcia - larilgarcia@hotmail.com \\ * Resident Physician in Pediatrics, General Hospital Roberto Santos, Salvador, BA, Brazil \\ ** Graduating in Medicine from Bahiana School of Medicine and Public Health, Salvador, BA, Brazil \\ *** Pediatrician, Member of the Brazilian Society of Pediatrics, Head of the Department of Pediatrics, General Hospital \\ Roberto Santos, Salvador, BA, Brazil
}

\begin{abstract}
Background: Splenic cysts are rare in children. They are classified into primary and secondary, also called pseudocysts. The primary feature capsule and can be congenital, vascular or neoclassical. The diagnosis, in most cases, is incidental because it is asymptomatic clinical status. The diagnosis is confirmed by Ultrasound and / or Computed Tomography (CT) of the abdomen. Case Report: Patient, I.T.S.A, female, 11 years old, complains of increasing abdominal size. The diagnosis of splenic cyst was confirmed with an exploratory laparotomy. The pathology suggests reactive mesothelial hyperplasia. The patient underwent a total splenectomy and recovered without complications. Conclusions: Splenic cysts are incidental findings, most of the times it has been an asymptomatic clinical status. In the clinical case described, the patient showed a significant increase in the abdomen. She underwent exploratory laparotomy and, consequently, total splenectomy, which is the standard treatment in cases of giants splenic cysts.
\end{abstract}

Kepwords: Pediatric Surgery; Splenic Cysts.

\section{INTRODUCTION}

Splenic cysts can be classified into type I (primary or true) or type II (secondary or pseudocysts). Type I cysts are encapsulated and can be of parasitic origin or not. The type I non-parasitic cysts can be congenital, vascular or neoplastic. Type II cysts have no capsule and is formed by fibrous tissue. (1) Splenic cysts larger than five centimeters are considered giants. From the radiological point of view, it is usually impossible to distinguish primary and secondary cysts.
Congenital splenic cysts or epithelial cysts comprise approximately $25 \%$ of true cysts of the spleen and have good prognosis. ${ }^{(2)}$ Although the exact mechanism of its pathogenesis is unknown, proposed mechanisms include: involution of pluripotent cells of the splenic parenchyma during development with squamous metaplasia. (1)

Macroscopic analysis typically shows a large, smooth, encapsulated cyst. Occasionally the cyst 
presents trabeculae or septa. Microscopically, the cyst is lined internally with columnar tissue, cuboid or squamous epithelium and can be subdivided into dermoid, epidermoid and mesothelial. (3)

Usually congenital splenic cysts are asymptomatic and have a good prognosis. However, in some cases, may become symptomatic due to the increase in the size. The diagnosis is often incidental during an examination of abdominal imaging. The number of diagnosed splenic cysts is probably growing due to the large amount of radiological examinations performed today.

\section{CASE REPORT}

Patient, I.T.S.A, female, 11 years old, born and raised in Salvador / BA, mulatto, incomplete primary education. Admitted to Hospital Geral
Roberto Santos due to a complaint of abdominal enlargement for 20 days. Patient reported that there are 20 days notices increased abdominal region especially the left, associated with dyspnea in the supine position, with improvement in orthostatic, mild pain, localized in the left upper quadrant, stabbing, self-limiting and no improvement or worsening factor. She had a history of constipation for about two years with two admissions to perform bowel cleansing without comorbidities.

On physical examination, distended abdomen due to a palpable mass in the left hypochondrium region, well-defined, smooth, painless on palpation, measuring about $25 \mathrm{~cm}$ in its largest diameter, which exceeds midline. Presents slight mobility and unchanged renal palpation. Massive sound on percussion of the left upper quadrant and bowel sounds present diffusely throughout the abdomen (Figure 1).

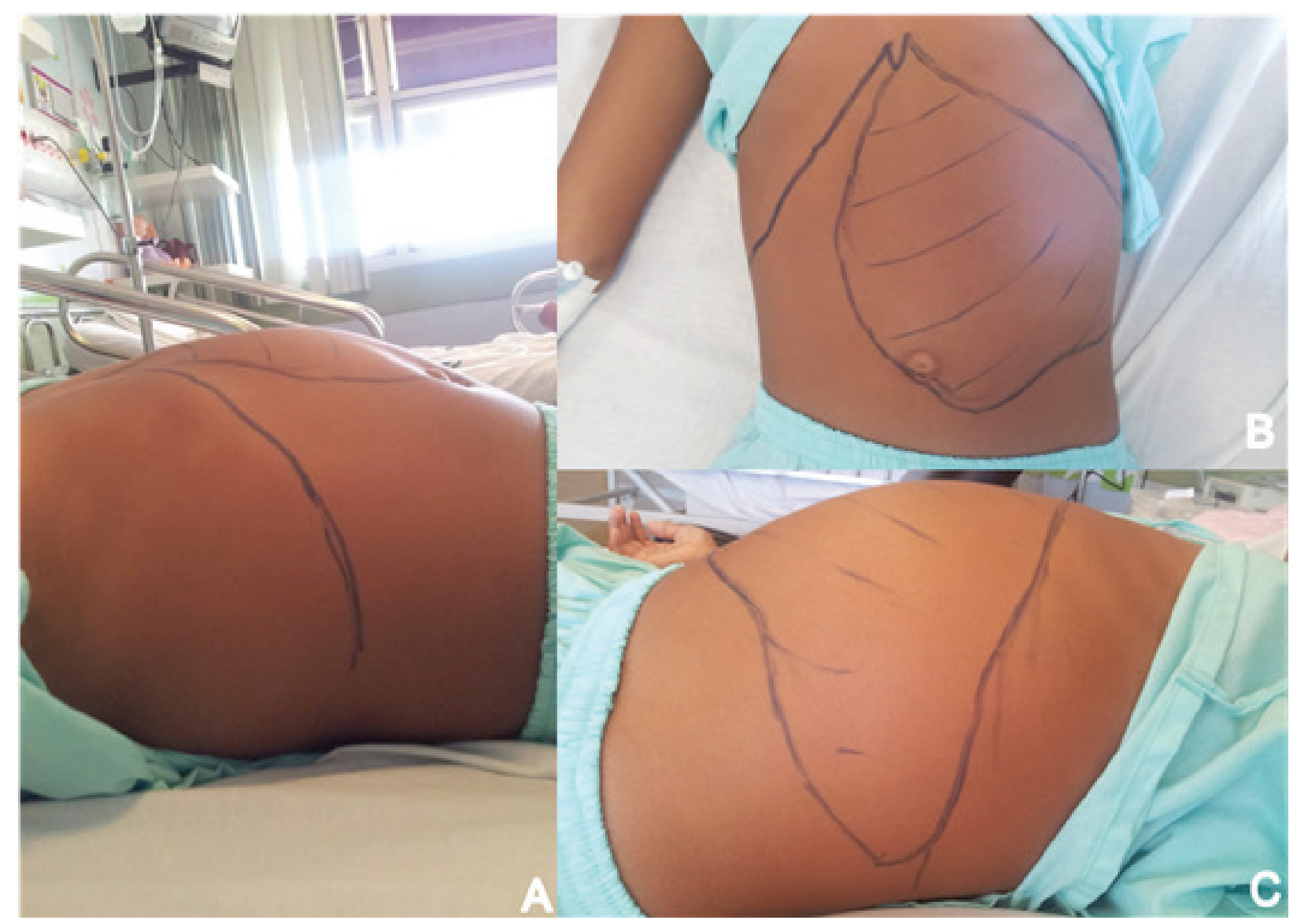

Figure 1 - Definition of abdominal mass and its anatomical relationship. A) Right lateral view. B) Front view in the supine position. C) Left lateral view 
On admission, patients' laboratory tests performed were normal: blood count, C-reactive protein, sodium, potassium and calcium serum alanine and aspartate aminotransferase, lactate dehydrogenase, alpha-fetoprotein, beta-HCG, CA125 and parasitological stool. Underwent abdominal ultrasonography revealed a mass that regular contour and well-defined limits, measuring approximately $13.4 \times 10.9 \mathrm{~cm}$ in left hypochondrium. No changes were seen on chest radiography in anteroposterior incidence. When performed total abdominal radiography, a hypotransparent image was observed in the left upper abdomen (Figure 2).
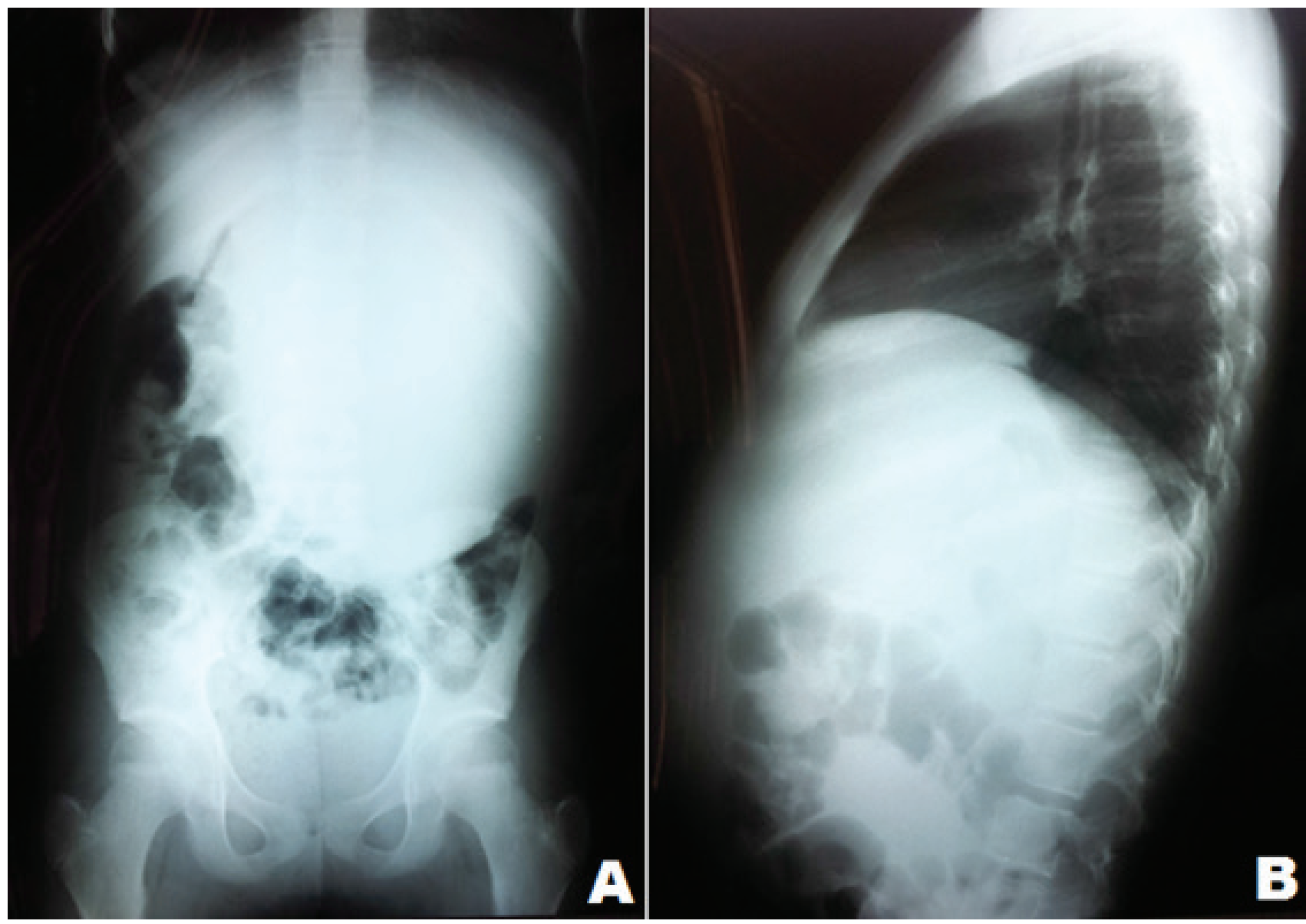

Figure 2 - total abdominal radiography. A) anteroposterior incidence. B) Incidence in profile

It was identified on CT bulky hypodense image in the left upper quadrant, with density of liquid. There are parietal calcifications, with apparent origin in the upper pole of the spleen measuring approximately $17.0 \times 12.0 \times 13.9 \mathrm{~cm}$, without contrast enhancement, determining inferior displacement of the spleen, medial of the stomach, hepatic left lobe and posterior of the pancreas (Figure 3 ). 

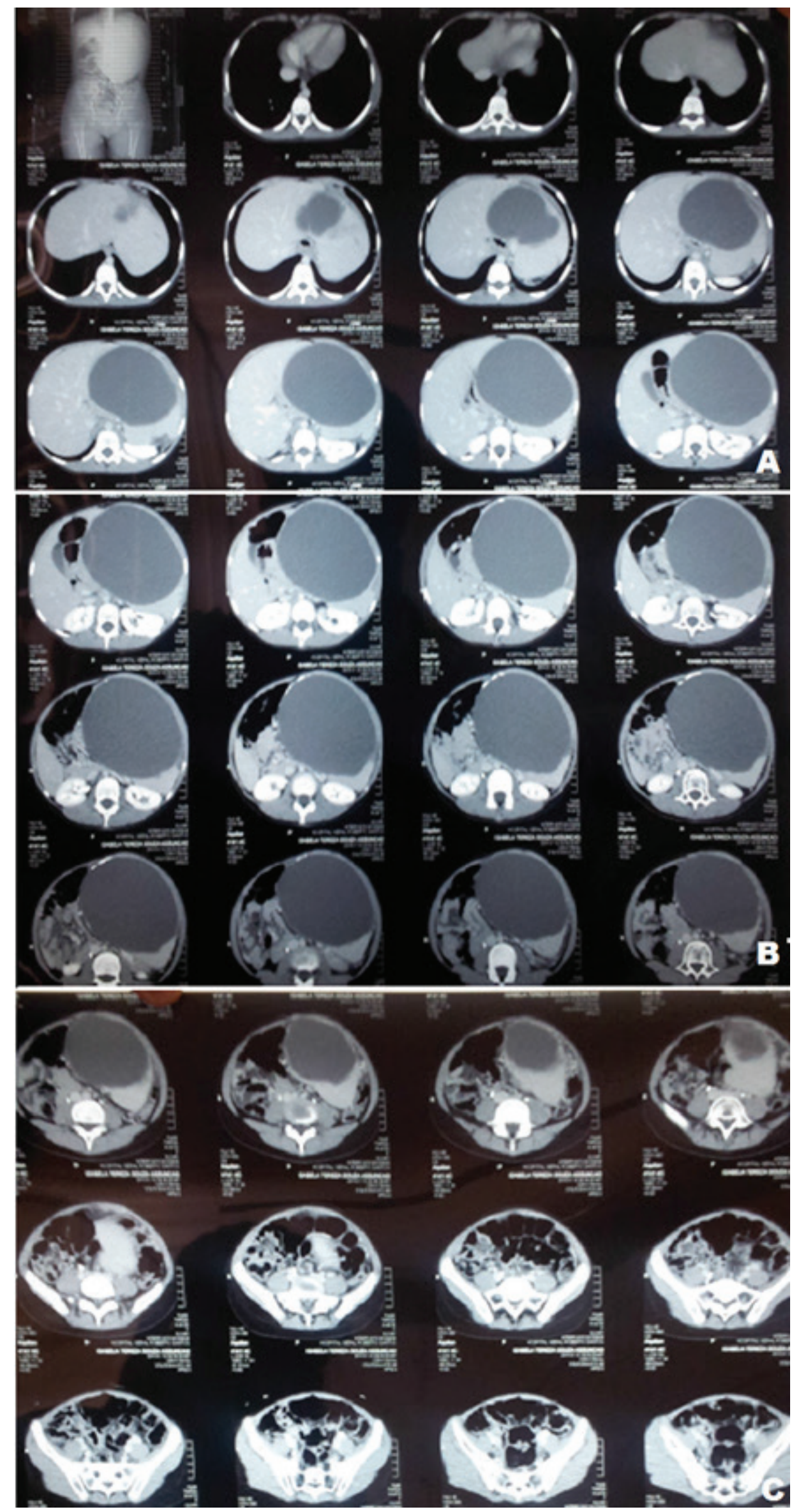

Figure 3 - Total abdominal CT. A) Mediastinum. B) Upper abdomen. C) Lower abdomen 
Patient underwent exploratory laparotomy, which revealed the presence of a splenic cyst. Then, it was performed total splenectomy with removal of the surgical specimen (Figure 4), which was sent to the cytology. There were no complications during surgery. Cytology was negative for neoplastic cells, consistent with reactive mesothelial hyperplasia.
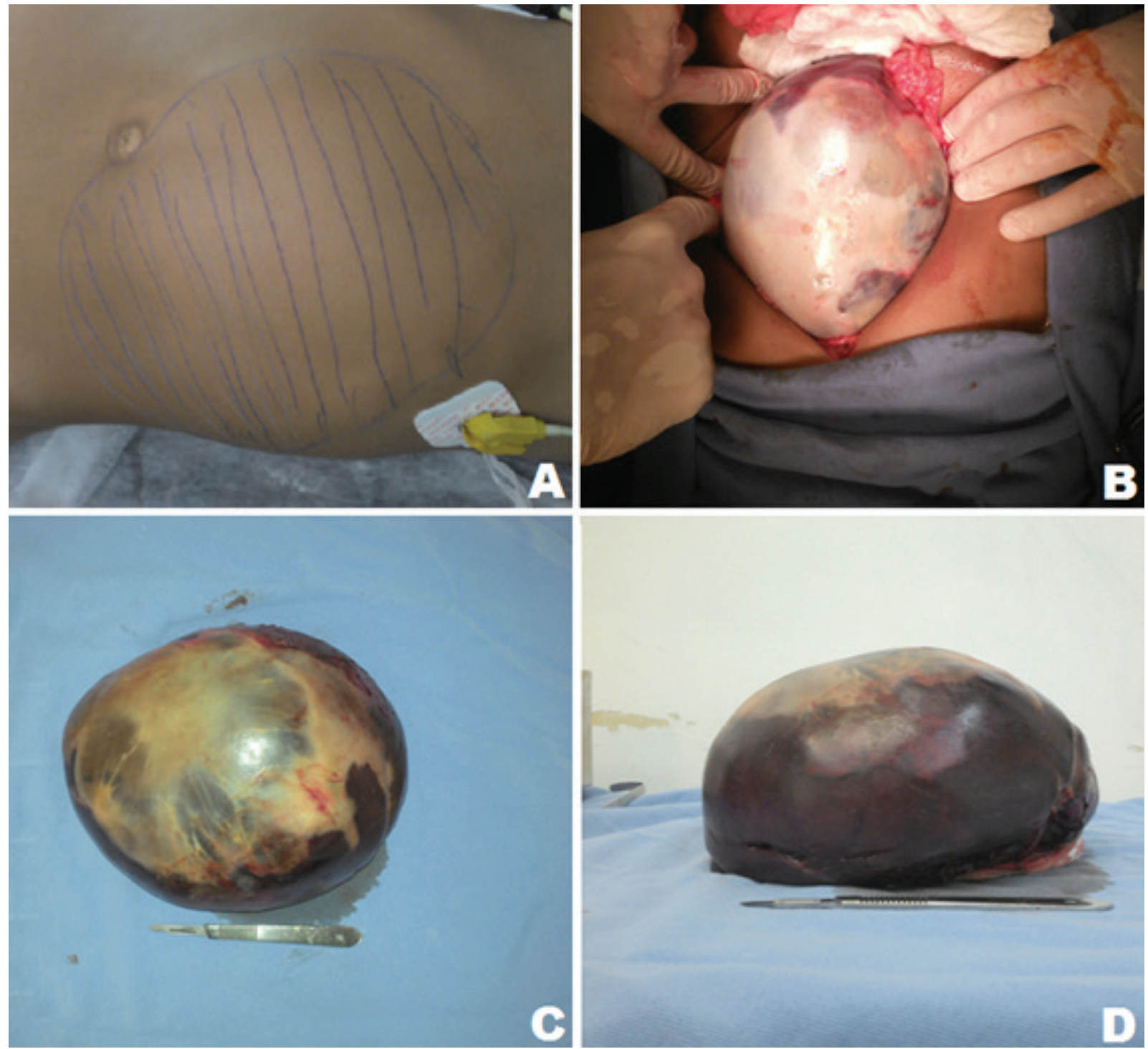

Figure 4 - A) Pre-operative image. B) Removal of the surgical specimen intraoperatively. C) Craniocaudal view of the surgical specimen. D) Front view of the surgical specimen

Patient developed clinically stable postoperatively without complications. Cefazolin was used for 48 hours. She persisted with constipation postoperatively, temporarily resolved with lactulona. Vaccination for haemophilus influenza B, pneumococcal 23 and tetravalent meningococcal were guided. Finally, the patient was discharged from hospital, monitored in a pediatric surgery.

\section{DISCUSSION}

Splenic cysts are more frequent in the second and third decades of life, but may appear in other age groups. ${ }^{(4)}$ Abdominal radiography may reveal a mass that may be calcified left upper quadrant. The ultrasound is able to differentiate solid and cystic lesions in most cases. Typically, it appears 
as a homogeneous mass, anechoic with thin walls. Septations, irregular walls or a heterogeneous pattern of internal echogenicity, with debris or hemorrhage and peripheral hyperechoic foci with posterior acoustic shadowing due to calcifications in the wall can be seen in the complex cysts. ${ }^{(1)}$

Computed tomography is more sensitive than ultrasound in identifying septa and calcifications. ${ }^{(5)}$ Cystic lesions on CT are spherical, well-defined, with attenuation equivalent to the water, with a thin or imperceptible capsule. ${ }^{(6)}$ Splenic cysts may contain areas with a high density due to bleeding, increased protein content, or purulent material into the cyst. ${ }^{(1)}$

A laparotomy with total splenectomy is recommended for giant splenic cysts or near the splenic hilum. However, more conservative surgical procedures, such as partial splenectomy - which preserves more than $25 \%$ of the splenic parenchyma - total cystectomy, marsupialization or cyst decapsulation currently have had greater applicability. Conservative treatment, especially in larger lesions, such as percutaneous aspiration or sclerosis, does not result in good long-term control. (2)

The main complications associated with surgery include infection, rupture and hemorrhage. (7) Preoperative immunization for meningococcus, pneumococcus and haemophilus should always be carried out with the aim of reducing the risk of infection. Some authors also employ prophylactic penicillin in the three months following the surgery, since the splenic function may take several months to recover to an immunologically competent level. ${ }^{\left({ }^{(8)}\right.}$

\section{CONCLUSION}

Splenic cysts are usually incidental findings, because in most cases are asymptomatic. In the clinical case described, the patient progressed with significant abdominal enlargement and underwent exploratory laparotomy and, consequently, total splenectomy which is the classical treatment in cases of giant splenic cysts. It is noteworthy that immunization was performed only postoperatively.

\section{ETHICAL ASPECTS}

Responsible for the patient signed an informed consent form authorizing the publication and reporting of the case, including photographs and exam results. The authors have ensured preserve the confidentiality of data and the sigil of patient identity.

\section{REFERENCES}

1. Schlittler LA, Dallagasperina VW. Cistos esplênicos não-parasitários. Rev. Col. Bras. Cir. 2010;37(6):442-446.

2. Hansen MB, Moller AC. Splenic cysts. Surg Laparosc Endosc Percutan Tech. 2004; 14: 316-22.

3. Giovagnoni A, Giorgi C, Goteri G. Tumours of the spleen. Cancer Imaging. 2005;5(1):73-7.

4. Mielle V, Galluzo M, Cortese A, Bellussi A, Valenti M. Diagnostic imaging of splenic cysts in children. Radiol Med. 1998; 95: 62-5.

5. Anon R, Guijarro J, Amoros C, Gil J, Bosca MM, Palmero J, Benages A. Congenital splenic cyst treated with percutaneous sclerosis using alcohol. Cardiovasc Intervent Radiol. 2006;29(4):691-3.

6. Ramani M, Reinhold C, Semelka RC, Siegelman ES, Liang L, Ascher SM et al. Splenic hemangiomas and hamartomas: MR imaging characteristics of 28 lesions. Radiology. 1997;202(1):166-72.

7. Trompetas V, Panagopoulos E, PriovolouPapaevangelou M, Ramantanis G. Giant benign true cyst of the spleen with high serum level of CA 19-9. Eur J Gastroenterol Hepatol. 2002;14(1):85-8.

8. Holland AJA, Ford WDA, Bourne AJ. Conservative surgery for benign non-parasitic splenic cysts. Pediatr Surg Int 1997; 12: 353-555. 